\section{J'TI}

JOURNAL OF

TRAUMA AND INJURY

Received: August 25, 2020

Revised: December 1, 2020

Accepted: December 9, 2020

\section{Correspondence to}

Jae-Woo Cho, M.D.

Department of Orthopedic Surgery, Korea

University Guro Hospital, 148 Guro-

dong-ro, Guro-gu, Seoul 08308, Korea

Tel: +82-2-2626-1869

E-mail:jeus1103@gmail.com

\section{Co-correspondence to}

Jong-Keon Oh, M.D.

Department of Orthopedic Surgery, Korea

University Guro Hospital, 148 Guro-

dong-ro, Guro-gu, Seoul 08308, Korea

Tel: +82-2-2626-1114

E-mail: jkoh@korea.ac.kr

\title{
The Effects of a Trauma Team Ap- proach on the Management of Open Extremity Fractures in Polytrauma Patients: A Retrospective Comparative Study
}

\author{
Seungyeob Sakong, M.D. ${ }^{1}$, Eic Ju Lim, M.D. ${ }^{1}$, Jun-Min Cho, M.D. ${ }^{2}$, \\ Nak-Jun Choi, M.D. ${ }^{2}$, Jae-Woo Cho, M.D. ${ }^{1}$, Jong-Keon Oh, M.D. ${ }^{1}$ \\ ${ }^{1}$ Department of Orthopedic Surgery, Korea University Guro Hospital, Seoul, Korea \\ ${ }^{2}$ Department of General Surgery, Korea University Guro Hospital, Seoul, Korea
}

Purpose: Open extremity fractures require prompt antibiotic medication and initial debridement surgery to reduce the infection rate and restore functional stabilization. We aimed to report the effects and positive outcomes of a trauma team approach on the management of open extremity fractures in polytrauma patients.

Methods: This retrospective review included all polytrauma patients with open extremity fractures admitted between March 2009 and December 2019. Patients were divided into two groups according to whether they were treated before or after the implementation of the trauma team approach (March 2014). We analyzed the outcomes in each group with respect to the time interval until the doctor's arrival, total length of stay in the emergency department, the time interval until initial antibiotic treatment and operation, whether the initial operation was performed within 24 hours, and the rate of deep infections.

Results: A total of 123 patients met the inclusion criteria. There were no statistically significant differences in demographic characteristics. The time interval until the doctor's arrival $(64.12 \pm 49.2$ minutes vs. $19.82 \pm 15.23$ minutes; $p=0.035)$ and initial antibiotic treatment ( $115.47 \pm 72.12$ minutes vs. $48.78 \pm 30.12$ minutes; $p=0.023)$ significantly improved after implementing the trauma team approach. The union rate was not significantly different. However, the time interval until initial debridement, opportunity for initial debridement within 24 hours, and the rate of deep infections demonstrated better results.

Conclusions: The reduced time interval until initial antibiotic treatment and debridement could be attributed to the positive effect of the trauma team approach on the management of open extremity fractures in polytrauma patients.

Keywords: Open fracture; Multiple trauma; Trauma team approach 


\section{INTRODUCTION}

More than $50 \%$ of patients with multiple trauma experience significant extremity injuries. Of these, $12 \%$ are diagnosed as open extremity fractures. These fractures are typically caused by high-energy blunt trauma and traffic accidents and include a combination of head, thorax, and abdomen trauma [1]. The risk of clinical infection from an open fracture depends on the severity of the injury and ranges from $0 \%$ to $2 \%$ for type I, $2 \%$ to $5 \%$ for type II, and $5 \%$ to $50 \%$ for type III [2]. Musculoskeletal infections impose a substantial financial burden in terms of total health care expenditures, as treatment costs for patients with infections were reported to be approximately 5 to 6 times higher than those for uninfected patients [3]. Without prompt antibiotic treatment and initial surgical debridement, copious irrigation can increase the infection rate, impede fracture healing, and reverse functional stabilization. Therefore, the prompt administration of antibiotics and transfer of patients to the operating room on an urgent basis with a multidisciplinary approach are essential for managing open extremity fractures in polytrauma patients.

To promote quick and effective trauma management, in 2012, the Korean government established a trauma system and designated regional trauma centers; currently, 17 such centers have been designated [4]. Several studies have reported that management by a trauma team can reduce both the mortality rate of trauma patients and the length of stay in the emergency department (ED) [5,6]. In March 2014, Korea University Guro Hospital established a specialized trauma team and initiated an urgent treatment processing system (UTPS). The following year, Korea University Guro Hospital was designated as a focused training center for trauma with support provided by the government.

Before establishment of the trauma team, the initial evaluation of open extremity fractures in polytrauma patients was conducted by ED clinicians. In that system, junior residents from a related department initially saw the patient and then eventually notified the chief residents and on-duty staff members. Rather than providing a coordinated evaluation with other departments during the initial management, each department was consulted after admission. Furthermore, because anesthesiologists were not notified during the ED admission process, it was impossible to prepare the operating room in advance, making it more difficult to perform emergency surgery. Patient follow-up was similar to that of general university hospitals in Korea, which can delay initial management. There are still some hospitals that manage these patients in a single department.

Korea University Guro Hospital reported positive effects of the trauma team approach for the management of hemodynamically unstable pelvic bone fractures in the orthopedic field. An overall positive improvement was shown in total length of ED stay, 24-hour mortality, and time interval until definitive fixation [7].

To date, no published studies have compared the trauma team approach with department-specific management of open extremity fractures in polytrauma patients. Therefore, we aimed to evaluate the effects of implementing a trauma team approach on the management of open extremity fractures in polytrauma patients.

\section{METHODS}

\section{Patients and Methods}

A focused training center for trauma was established at Korea University Guro Hospital in March 2014. At that time, the specialized trauma team and trauma UTPS were implemented. Patients were divided into two groups according to when they were admitted by referring to their medical records. Patients in group A were admitted from March 2009 to February 2014, and patients in group B were admitted from March 2014 to December 2019, after the launch of the UTPS. The inclusion criteria of the subjects were as follows: 1) patients older than 18 years with open extremity fractures; 2) polytrauma patients with an Injury Severity Score (ISS) of $\geq 15$, based on the Abbreviated Injury Scale; and 3) treatment with an initial debridement operation with or without primary fracture stabilization for an open extremity fracture. The exclusion criteria were 1) patients with insufficient data for analysis; 2) a mechanism of injury that was either burn or penetrating trauma; 3) fractures to the spine, pelvis, hands, or feet; 4) patients who underwent an initial amputation operation for treat- 
ment or who arrived at the ED in an amputated state; and 5) patients who presented to the ED 6 hours or more after injury.

The following demographic variables were collected and evaluated in both groups: age, sex, open fracture classification (Gustilo-Anderson classification), ISS, Revised Trauma Scale, systolic blood pressure, mean arterial blood pressure, and injury mechanism.

For each group, we evaluated the time interval until the doctor's (attending's) arrival, time interval until the initial antibiotic treatment order (minutes), total length of ED stay (minutes), the amount of packed red blood cells transfused within 24 hours (units), the time interval until the initial operation (hours), and the rate of deep infections after surgery (defined as infections of the bone and deep tissue necessitating unplanned operative irrigation and debridement). Planned repetitive debridement and superficial infections that did not require further surgery were not considered to be deep infections.

\section{Imaging}

Plain anteroposterior and lateral radiographs were obtained postoperatively at 7-week intervals until the fracture was healed. Radiographs were evaluated by three trained reviewers who were not involved in the patients' care. Union was defined as the healing of at least three of four cortices on biplanar radiographs. Nonunion was defined as a fracture at least 9 months old that did not show any signs of healing for 3 consecutive months.

\section{Trauma team approach and activation}

The trauma team approach in Korea University Guro Hospital was initiated in March 2014 and involves experienced specialists from the ED, trauma surgery, orthopedic, chest surgery, neurosurgery, plastic surgery, and urological surgery departments.

The criteria for the trauma UTPS include the following: 1) systolic blood pressure of $<90 \mathrm{mmHg}$ in adults, 2) intubation requirement or transferred with intubation, 3) body trunk or extremity injuries with a Glasgow Coma Scale score of $<13,4$ ) unstable pelvic bone fracture, 5) degloving injuries, 6) multiple fractures, 7) penetrating neck or trunk injuries, and 8) fall from a height of $>5 \mathrm{~m}$ in adults.
The UTPS is activated when a patient meets the specified criteria. These patients appear in red letters on the order communication system, and a pop-up text box displays the text "Trauma UTPS". Labeling these patients in red enables them to be recognized in the X-ray, computed tomography, and magnetic resonance imaging rooms, angiography rooms, operating rooms, and intensive care units, so that patients can be prepared and receive diagnosis and treatment as soon as possible, reflecting their prioritization over ordinary patients. Brief information, including the patient's ID, related inclusion criteria for UTPS designation, and diagnosis, is sent to the trauma team members' cellular phones via SMS. Primary resuscitation is performed by the department of trauma surgery immediately after UTPS activation. Subsequently, a secondary survey by the corresponding departments should be performed within 30 minutes.

\section{Treatment protocol}

We initiate intravenous antibiotic treatment upon presentation, and continue antibiotic treatment until 24 hours after the initial surgical intervention. Cefazolin ( $1 \mathrm{~g}$ intravenously) every 8 hours is administered for Gustilo-Anderson grade I patients, and intravenous gentamicin (with weight-adjusted dosing) or levofloxacin (500 mg every 24 hours) is added for patients with Gustilo-Anderson II and III fractures.

Initial debridement is undertaken urgently based on the availability of an operating theater. The timing of wound closure and the method of fixation are left to the discretion of the surgeon.

\section{Statistical analysis}

We used SPSS version 20.0 (IBM Corp., Armonk, NY, USA) for statistical analysis. The independent $t$-test, chisquare test, and Fisher exact test were performed for comparisons between the two groups. For all tests, $p$-values $<0.05$ was considered to indicate statistical significance.

\section{RESULTS}

\section{Demographic data}

A total of 123 patients met the inclusion criteria between 
January 2009 and December 2019. In group A, 53 patients were enrolled (two with fractures of the humerus, three

Table 1. Demographic data and trauma scores

\begin{tabular}{|lccc|}
\hline & Before $(\mathbf{n}=53)$ & After $(\mathbf{n}=70)$ & $p$-value \\
\hline Age (years) & $57.24 \pm 18.21$ & $58.31 \pm 20.73$ & $0.375^{\mathrm{a}}$ \\
Sex (male/female) & $38 / 15$ & $53 / 17$ & $0.324^{\mathrm{b}}$ \\
Open fracture type (G-A) & & & $0.235^{\mathrm{c}}$ \\
1 & 6 & 8 & \\
2 & 27 & 34 & \\
3A & 13 & 16 & \\
3B & 5 & 7 & \\
3C & 2 & 5 & \\
Injury of mechanism & & & $0.508^{\mathrm{c}}$ \\
Traffic accident & 42 & 60 & \\
Fall from $>3$ m & 5 & 6 & \\
Fall from $<3 \mathrm{~m}$ & 1 & 1 & \\
Others & 5 & 3 & \\
Initial SBP (mmHg) & $87.57 \pm 30.8$ & $86.58 \pm 31.3$ & $0.685^{\mathrm{a}}$ \\
Initial MAP (mmHg) & $70.38 \pm 25.5$ & $68 \pm 23.7$ & $0.712^{\mathrm{a}}$ \\
RTS & $5.43 \pm 0.78$ & $5.51 \pm 0.73$ & $0.887^{\mathrm{a}}$ \\
ISS & $22.84 \pm 12.34$ & $23.92 \pm 13.37$ & $0.875^{\mathrm{a}}$ \\
\hline
\end{tabular}

G-A: Gustilo-Anderson, SBP: systolic blood pressure, MAP: mean arterial pressure, RTS: Revised Trauma Score, ISS: Injury Severity Score.

andependent $t$-test.

${ }^{\mathrm{b}}$ Chi-square test.

'Fisherman's test. with fractures of the radius, one with a fracture of the ulna, 17 with fractures of the femur, and 30 with fractures of the tibia). In group B, 70 patients were enrolled (five with fractures of the humerus, four with fractures of the radius, two with fractures of the ulna, 22 with fractures of the femur, 33 with fractures of the tibia, and four with fractures of the femur and tibia). There were no statistically significant differences in demographic data including mean age, sex, and open fracture classification (Gustilo-Anderson system). Gustilo-Anderson type II was the most common type of open fracture in both groups, and traffic accidents were the most common cause of injury. No statistically significant differences were found between the groups in systolic blood pressure, mean arterial blood pressure, ISS, and Revised Trauma Scale scores (Table 1).

\section{Outcome data}

The time intervals until the doctor's (attending's) arrival (64.12 \pm 49.2 minutes vs. $19.82 \pm 15.23$ minutes; $p=0.035$ ) and initial antibiotic medication order (115.47 \pm 72.12 minutes vs. $48.78 \pm 30.12$ minutes; $p=0.023)$ were significantly shorter in group B than in group A. The total length of ED stay (256.32 \pm 62.12 minutes vs. $114.34 \pm 48.28$ minutes; $p=0.025$ ) was also significantly shorter in group B. However, the amount of packed red blood cells transfused within 24 hours was not significantly different between the two groups.

A significantly shorter time interval was found in

Table 2. Comparison of outcomes

\begin{tabular}{|c|c|c|c|}
\hline & Before $(n=53)$ & After $(n=70)$ & $p$-value \\
\hline Time interval for doctor's arrival (min) & $64.12 \pm 49.2$ & $19.82 \pm 15.23$ & $0.035^{\mathrm{a}}$ \\
\hline Time interval for initial antibiotics medication's order (min) & $115.47 \pm 72.12$ & $48.78 \pm 30.12$ & $0.023^{\mathrm{a}}$ \\
\hline Total length of staying in ED (min) & $256.32 \pm 62.12$ & $114.34 \pm 48.28$ & $0.025^{\mathrm{a}}$ \\
\hline 24-hour transfusion of packed RBCs (unit) & $3.78 \pm 2.34$ & $4.21 \pm 2.18$ & $0.859^{\mathrm{a}}$ \\
\hline Time interval for initial debridement operation (hours) & $32.32 \pm 10.21$ & $23.87 \pm 7.17$ & $0.002^{\mathrm{a}}$ \\
\hline Initial debridement in 24 hours (n \%) & $49.1(26 / 53)$ & $77.1(55 / 70)$ & $0.001^{b}$ \\
\hline Union rate (\%) & $90.5(48 / 53)$ & $94.2(66 / 70)$ & $0.352^{b}$ \\
\hline Rate of deep infection after surgery (\%) & $13.2(7 / 53)$ & $2.8(2 / 70)$ & $0.031^{b}$ \\
\hline Rate of deep infection after surgery in G-A type 3 & $25(5 / 20)$ & $7.14(2 / 28)$ & $0.11^{b}$ \\
\hline
\end{tabular}

ED: emergency department, RBC: red blood cell, G-A: Gustilo-Anderson.

andependent $t$-test.

bFisherman's test. 
group B for the time interval until initial debridement (32.32 \pm 10.21 hours vs. $23.87 \pm 7.17$ hours; $p=0.002)$. The proportion of patients who underwent initial surgical debridement within 24 hours was significantly higher in group B ( $49.1 \%$ vs. $77.1 \%$; $p=0.001)$, whereas the proportion of those with deep infections was significantly lower in group B ( $13.2 \%$ vs. $2.8 \%$; $p=0.031)$. The proportion of deep infections in patients with Gustilo-Anderson type III open fractures in group A was more than three times higher than that in group B ( $25 \%$ vs. $7.14 \%$; $p=0.11)$, but the difference was not statistically significant. The union rate was also not significantly different between the two groups (Table 2).

\section{DISCUSSION}

The most significant result of this study is that without additional medical devices or personnel, we were able to perform more effective and prompt treatment for polytrauma patients with open extremity fractures by modifying the previous system and establishing a trauma team approach. As a result, the rate of deep infections has been reduced.

However, although the time interval until initial debridement significantly decreased after UTPS application, it was still twice as long as has been reported in other countries' trauma centers. Hull et al. [8] reported a much shorter time interval until initial debridement (10.6 hours) in a level 1 trauma hospital in North America. A study from 3 level 1 Canadian trauma centers also reported an interval of 9.1 hours until initial debridement [9].

Originally, without the UTPS system, the initial evaluation for open extremity fractures in patients with multiple trauma was performed by on-duty junior residents following the intern's notification. Unfortunately, those on-duty residents could not focus only on patients in the ED because they had many duties in wards, operating rooms, and outpatient clinics. Furthermore, more senior residents and specialists were usually notified after a full radiologic evaluation, which could further delay the final notification.

Since the establishment of a specialized trauma team, the trauma coordinator has served as a direct contact with the trauma team including orthopedic attendings, instead of a complicated notification system; therefore, more prompt and accurate initial diagnosis and management for open extremity fractures in polytrauma patients could be achieved.

The length of stay in the ED of patients with multiple injuries can be prolonged in many cases because of complicated treatment processes and diagnoses $[10,11]$. Furthermore, difficulty in deciding which department is primarily responsible for the patient exacerbates this delay.

However, after implementing the trauma team approach, specialists from each department became involved in the initial management of patients in the ED, which enabled direct communication with other related departments.

Antibiotic treatment has been considered the standard of care for managing open fractures since 1974, when first reported by Patzakis et al. [12], Gosselin et al. [13] showed that the administration of antibiotics after an open fracture reduced the risk of infection by 59\%. Many studies have suggested the necessity of rapid antibiotic administration after injury in open fracture patients and demonstrated its efficacy [14-16].

Antibiotic treatment previously tended to be delayed or ignored because of the junior residents' lack of experience and focus on diagnosis and dressing. Upon admission to other departments, delayed orders for antibiotic medication can easily occur. After initiating the trauma team approach, the orthopedic attending was notified immediately upon the patient's arrival, and antibiotic treatment was promptly initiated to reduce the risk of infection.

Traditionally, debridement of an open fracture within 6 hours of injury (i.e., the so-called 6-hour rule) has been recommended [17-19]. Hull et al. [8] reported that delayed debridement of severe open fractures (GustiloAnderson grades II and III) was associated with a linear increase in the rate of deep infections (3\% per hour of delay). Conversely, several studies have called the 6-hour rule into question [20-22]. However, those studies were not randomized and not adequately powered to detect clinically meaningful differences in infection rates, as they had small sample sizes [2]. Schenker et al. [23] identified there was no evidence to support the 6-hour rule. However, they also concluded that a purposeful delay of 
treatment in patients with open fractures is not recommended, and any delay should be minimized. Therefore, open fractures should be treated urgently with surgical debridement as a principle.

After initiating the trauma team approach, both primary resuscitation by specialists from related department and cooperation with anesthesiologists to request emergency surgery became much more effective. When the UTPS is activated, this information is also shared with the anesthesia department, and the on-duty anesthesiologist begins to prepare the operation room and scrub nurses for emergency surgery. Furthermore, the hospital is equipped with two dedicated surgery rooms for only trauma patients. As a result of these effective systems, we have been able to shorten the time interval until initial debridement to a remarkable extent, and more than $70 \%$ of patients now receive surgery within 24 hours, as compared with fewer than $50 \%$ before UTPS activation.

For the treatment of open fractures, the surgeon's objectives are to prevent infection and promote fracture healing. Infections after open fractures increase treatment complexity, affect patients' outcomes, and increase morbidity $[24,25]$. Deep infections are significantly associated with delayed healing and nonunion after long bone fractures [9]. Increased numbers of unplanned debridement operations after an improper initial response for open fracture have considerable implications for the patient's functional capacity and quality of life, as well as health care resource demands. Therefore, proper treatment to prevent infection and additional unplanned debridement operations are closely related to the prognosis.

Because more effective and rapid treatment for open extremity fractures could be delivered after initiating the trauma team approach, the rate of deep infections and the likelihood of unplanned debridement operations were reduced.

We expected more favorable results in terms of the rate of union. However, the data showed no significant difference between the two groups. Regardless of the improved time interval for initial antibiotic medication and debridement, adequate surgical management with high-quality reduction is another important factor that can affect the rate of union.

There are several limitations to our study. First, this was a retrospective study with a small sample size, meaning that the findings could be influenced by unexpected factors. Second, we could not evaluate whether functional scores revealed improvements in outcomes. Third, because of incomplete data, additional factors that had not been recorded systematically may have been omitted. Fourth, this study was limited to patients treated at a single representative hospital.

Our study is significant because of the following points. First, it is the first study to investigate the utility of a trauma team approach for open extremity fractures in polytrauma patients. Second, the effectiveness of this system was clarified, suggesting the need for its active introduction in the field of orthopedics. Third, without additional in terms of medical resources or facilities, we were able to achieve these improvements only by modifying the treatment pathway and implementing systemic cooperation with other departments. Fourth, because the time interval until the first debridement operation was still twice as long as reported for other countries' trauma centers, more favorable results could be achieved by establishing a standardized trauma management protocol and expanding the operating range of the trauma team approach and facilities such as the intensive care unit and operating room with active support from the government.

\section{CONCLUSION}

The trauma team approach had positive effects on the management of open extremity fractures in multiple trauma patients. The introduction of the trauma team approach system resulted in improvements in initial resuscitation with multidisciplinary decision-making, a shorter time until initial antibiotic treatment and surgical debridement, and a decreased rate of deep infections as compared with the previous system.

\section{REFERENCES}

1. Banerjee M, Bouillon B, Shafizadeh S, Paffrath T, Lefering R, Wafaisade A; German Trauma Registry Group. Epidemiology of extremity injuries in multiple trauma patients. Injury 
2013;44:1015-21.

2. Okike K, Bhattacharyya T. Trends in the management of open fractures. A critical analysis. J Bone Joint Surg Am 2006;88:273948.

3. Metsemakers WJ, Smeets B, Nijs S, Hoekstra H. Infection after fracture fixation of the tibia: analysis of healthcare utilization and related costs. Injury 2017;48:1204-10.

4. Shim H, Jang JY, Lee JG, Kim S, Kim MJ, Park YS, et al. Application of critical pathway in trauma patients. J Trauma Inj 2012;25:159-65.

5. Khetarpal S, Steinbrunn BS, McGonigal MD, Stafford R, Ney AL, Kalb DC, et al. Trauma faculty and trauma team activation: impact on trauma system function and patient outcome. J Trauma 1999;47:576-81.

6. Lee SH, Cho SJ, Yeom SR, Ryu JH, Jung JW, Han SK, et al. Effect of the emergency trauma team's management on the treatment of patients with multiple severe trauma. J Korean Soc Traumatol 2009;22:172-8.

7. Cho WT, Cho JW, Kim J, Kim JK, Oh JK, Kim HJ, et al. The effect of trauma team approach on the management of hemodynamically unstable pelvic bone fracture: retrospective comparative study. J Trauma Inj 2016;29:139-45.

8. Hull PD, Johnson SC, Stephen DJ, Kreder HJ, Jenkinson RJ. Delayed debridement of severe open fractures is associated with a higher rate of deep infection. Bone Joint J 2014;96-B:379-84.

9. Westgeest J, Weber D, Dulai SK, Bergman JW, Buckley R, Beaupre LA. factors associated with development of nonunion or delayed healing after an open long bone fracture: a prospective cohort study of 736 subjects. J Orthop Trauma 2016;30:14955.

10. Song BH, Hyun SY, Kim JJ, Cho JS, Ma DS, Kim HK, et al. A comparison of the effectiveness of before and after the regional trauma center's establishment. J Trauma Inj 2016;29:68-75.

11. Kang MJ, Shin TG, Sim MS, Jo IJ, Song HG. Factors affecting the delay of a decision to admit severe trauma patients and the effect of a multidisciplinary department system: a preliminary study. J Korean Soc Traumatol 2010;23:113-8.

12. Patzakis MJ, Harvey JP Jr, Ivler D. The role of antibiotics in the management of open fractures. J Bone Joint Surg Am 1974;56:532-41.
13. Gosselin RA, Roberts I, Gillespie WJ. Antibiotics for preventing infection in open limb fractures. Cochrane Database Syst Rev 2004;(1):CD003764.

14. Patzakis MJ, Wilkins J. Factors influencing infection rate in open fracture wounds. Clin Orthop Relat Res 1989;(243):36-40.

15. Obremskey W, Molina C, Collinge C, Nana A, Tornetta P 3rd, Sagi C, et al. current practice in the management of open fractures among orthopaedic trauma surgeons. Part A: initial management. A survey of orthopaedic trauma surgeons. J Orthop Trauma 2014;28:e198-202.

16. Zalavras CG, Marcus RE, Levin LS, Patzakis MJ. Management of open fractures and subsequent complications. J Bone Joint Surg Am 2007;89:884-95.

17. Robson MC, Duke WF, Krizek TJ. Rapid bacterial screening in the treatment of civilian wounds. J Surg Res 1973;14:426-30.

18. Kindsfater K, Jonassen EA. Osteomyelitis in grade II and III open tibia fractures with late debridement. J Orthop Trauma 1995;9:121-7.

19. Kreder HJ, Armstrong P. A review of open tibia fractures in children. J Pediatr Orthop 1995;15:482-8.

20. Harley BJ, Beaupre LA, Jones CA, Dulai SK, Weber DW. The effect of time to definitive treatment on the rate of nonunion and infection in open fractures. J Orthop Trauma 2002;16:484-90.

21. Ashford RU, Mehta JA, Cripps R. Delayed presentation is no barrier to satisfactory outcome in the management of open tibial fractures. Injury 2004;35:411-6.

22. Charalambous CP, Siddique I, Zenios M, Roberts S, Samarji R, Paul A, et al. Early versus delayed surgical treatment of open tibial fractures: effect on the rates of infection and need of secondary surgical procedures to promote bone union. Injury 2005;36:656-61.

23. Schenker ML, Yannascoli S, Baldwin KD, Ahn J, Mehta S. Does timing to operative debridement affect infectious complications in open long-bone fractures? A systematic review. J Bone Joint Surg Am 2012;94:1057-64.

24. Yusof NM, Halim AS. Outcomes of infected grade IIIB open tibial fractures. Singapore Med J 2012;53:591-4.

25. Patzakis MJ, Zalavras CG. Chronic posttraumatic osteomyelitis and infected nonunion of the tibia: current management concepts. J Am Acad Orthop Surg 2005;13:417-27. 